\title{
The skin-to-calyx distance measured by renal ct scan and ultrasound
}

Chen Jen Shan, Eduardo Mazzucchi, Fabio Payão, Andrea Cavalanti Gomes, Ronaldo Hueb Baroni, Fabio Cesar Torricelli, Fabio Carvalho Vicentini, Miguel Srougi

Division of Urology (CJS, MS), Section of Endourology (EM, FCT, FCV) and Division of Radiology (FP, ACG, RHB), Hospital das Clínicas, University of Sao Paulo Medical School, Sao Paulo, Brazil

\section{ABSTRACT}

Purpose: We developed a stereotactic device to guide the puncture for percutaneous nephrolithotripsy, which uses the distance from the target calyx to its perpendicular point on skin (SCD) to calculate the needle's entry angle. This study seeks to validate the use of measurements obtained by ultrasound (US) and computerized tomography (CT) for needle's entry angle calculation and to study factors that may interfere in this procedure.

Materials and Methods: Height, weight, abdominal circumference, CT of the urinary tract in dorsal decubitus (DD) and ventral decubitus (VD), and US of the kidneys in VD were obtained from thirty-five renal calculi patients. SCD obtained were compared and correlated with body-mass index (BMI).

Results: BMI was $28.66 \pm 4.6 \mathrm{Kg} / \mathrm{m}^{2}$. SCD on CT in DD was $8.40 \pm 2.06 \mathrm{~cm}$, in VD was $8.32 \pm 1.95 \mathrm{~cm}$, in US was $6.74 \pm 1.68 \mathrm{~cm}$. SCD measured by US and CT were statistically different $(\mathrm{p}<0.001)$, whereas between CT in DD and VD were not. SCD of the lower calyx presented moderate correlation with BMI.

Conclusion: SCD obtained by CT in ventral and dorsal decubitus may be used for calculation of the needle's entry angle. SCD obtained by US cannot be used. A rule for the correlation between BMI and the SCD could not be determined.

\section{ARTICLE INFO}

Key words:

Urolithiasis; Nephrostomy, Percutaneous; Radiosurgery;

Body Mass Index

Int Braz J Urol. 2014; 40: 212-9

Submitted for publication:

March 07, 2013

Accepted after revision:

March 19, 2014

\section{INTRODUCTION}

Surgical treatment of renal stones presented considerable advances during recent decades. Before the 70s, retained stones were removed surgically by means of large incisions in the skin. After that decade, the treatment of calculi came to be undertaken by extracorporeal shockwave lithotripsy (ESWL) (1), percutaneous nephrolithotripsy (PCNL) (2-4) and endoscopic ureterolithotripsy. Today PCNL is the gold standard treatment for renal stones bigger than $2 \mathrm{~cm}$ (5).
PCNL begins with an ultrasound or fluoroscopy guided puncture directed toward the desired renal calyx for removal of the stone. In some cases various attempts are necessary before the calyx is successfully reached, even by experienced surgeons, as the guidance given by fluoroscopy is made on a single plane (6). In up to $9 \%$ of cases it is impossible to undertake the procedure as a result of inability to attain the calyx (7). Only 27\% of the urologists trained in PCNL during their medical internship continue to carry out the procedure of renal access by percutaneous approach. 
The main reason is the technical difficulties of the method (8). An adequate puncture that penetrates the calyx's fornix is fundamental, as it diminishes the lesion of vessels and increases the chances of the patient's becoming stone-free $(9,10)$. For the purpose of accessing the renal calyx more easily, we have been developing a stereotactic localization system, an apparatus called the "Renal Puncturometer". With this device it is possible to determine the exact position of the calyx and to guide the needle directly towards the desired calyx, based on the distance between the calyx and the point on the skin perpendicular to the calyx to be punctured. We have called this measurement the Skin-to-Calyx Distance (SCD). The measurement of this distance has not been standardized and there is no data regarding if this distance measured by computerized tomography (CT) or ultrasound (US) is the same.

Our purposes were to study the factors which may alter the skin-to-calyx distance, particularly the decubitus position and the body mass index (BMI) and to evaluate the feasibility of using computerized tomography or ultrasound to estimate the Skin-to-calyx Distance (SCD).

\section{MATERIALS AND METHODS}

This study was approved by the Ethics Committee for the Analysis of Research Projects of the University of Sao Paulo Medical School, under $\mathrm{n}^{\circ}$ 1348/09. After consultation with the urologist, patients who agreed to participate in the study gave written informed consent. Between May 2010 and April 2011, thirty five patients diagnosed with renal calculi were included in the project. Age ranged from 18 to 65 years old. Pregnant women and patients with one kidney were excluded from the study.

Measurements of height, weight, abdominal circumference at the xiphoid appendix and at the umbilical scar were obtained. Non-contrast enhanced helicoidal CT was obtained for each patient in the dorsal decubitus (DD) and ventral decubitus (VD) and US of the kidneys in the ventral decubitus (VD) was also obtained. To perform the VD exams, $10 \mathrm{~cm}$ diameters bolsters were placed at the level of the shoulder joints and at the abdo- men. In order to reduce radiation exposure, the CT in VD was restricted to the kidney region.

The working principle of the device is to obtain a right-angle triangle (Figure-1) formed by the target calyx (C), the point on the skin in the lumbar region which is the perpendicular projection of the target calyx (S) and the projection of the needle's entry point on the skin (E) on S' plane (E projection). In a right-angle triangle, when we know the length of the two sides, we can calculate the angles and the hypotenuse (CE projection). One of the two sides is the distance between the projection of the point on the skin at which the needle entry on the S' plan (E projection) and the point on the skin perpendicular to the target calyx (SE). The other side is the distance between the target calyx and the point on the skin which is its perpendicular projection (SC), which is the distance under study here. For the purpose of clarity we will call it skin-to-calyx distance (SCD).

Figure-2 shows the smaller triangle in red, which is derived from the bigger triangle in green. The smaller triangle (in red) allows us to calculate the needle's entry angle. The identification of these triangles is cornerstone to the utilization of the device we are developing. We plan to validate that our calculations allow accurate targeting of the calyx in our next study by applying this process in PCNL.

The distances between the posterior calyx and the skin on the lumbar region perpendicular to the calyx (SCD) were obtained from the exams undertaken (US in VD and CT in VD and DD). We compared the distances obtained by the three exams and their correlation with the body-mass index (BMI) and abdominal circumference.

\section{Statistical analysis}

The values obtained were expressed in absolute values, average and standard deviation. Measures were compared using Analysis of Variance for repeated measurements $(11,12)$. A level of significance of 5\% ( $<$ 0.05) was employed for this assessment.

Correlation between the distances and the measurements of the abdominal circumference and body-mass index (BMI) were undertaken by means of Pearson's correlation coefficient $(11,12)$. 
Figure 1 - Drawing of the right-angle triangle: SC (Skin to Calix Distance) - Distance from the target calyx (C) to its perpendicular projection on the skin (S). SE - Distance between the projection of the point at which the needle enters the skin on S plane (E projection) and the point on the skin perpendicular to the target calyx (S). CE projection - Distance from the projection of the point at which the needle enters the skin on S plane (E projection) to the target calyx (C).

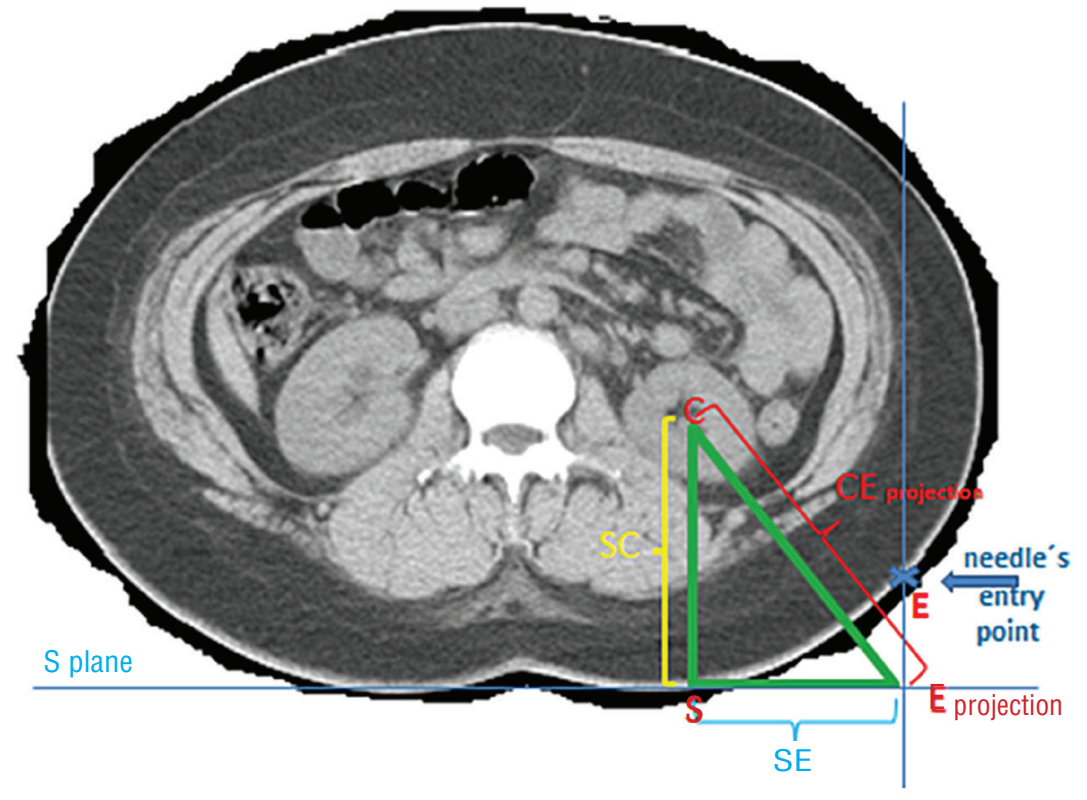

Figure 2 - Drawing of the smaller right-angle triangle in red: SC-N - Distance from the target calyx (C) to its perpendicular projection on the skin (S) minus the distance beetween E plane and S plane. SE - Distance between the needle's entry point on the skin (E) and SC line, forming with it a right angle. EC - Distance from the point at which the needle enters the skin to the target calyx.

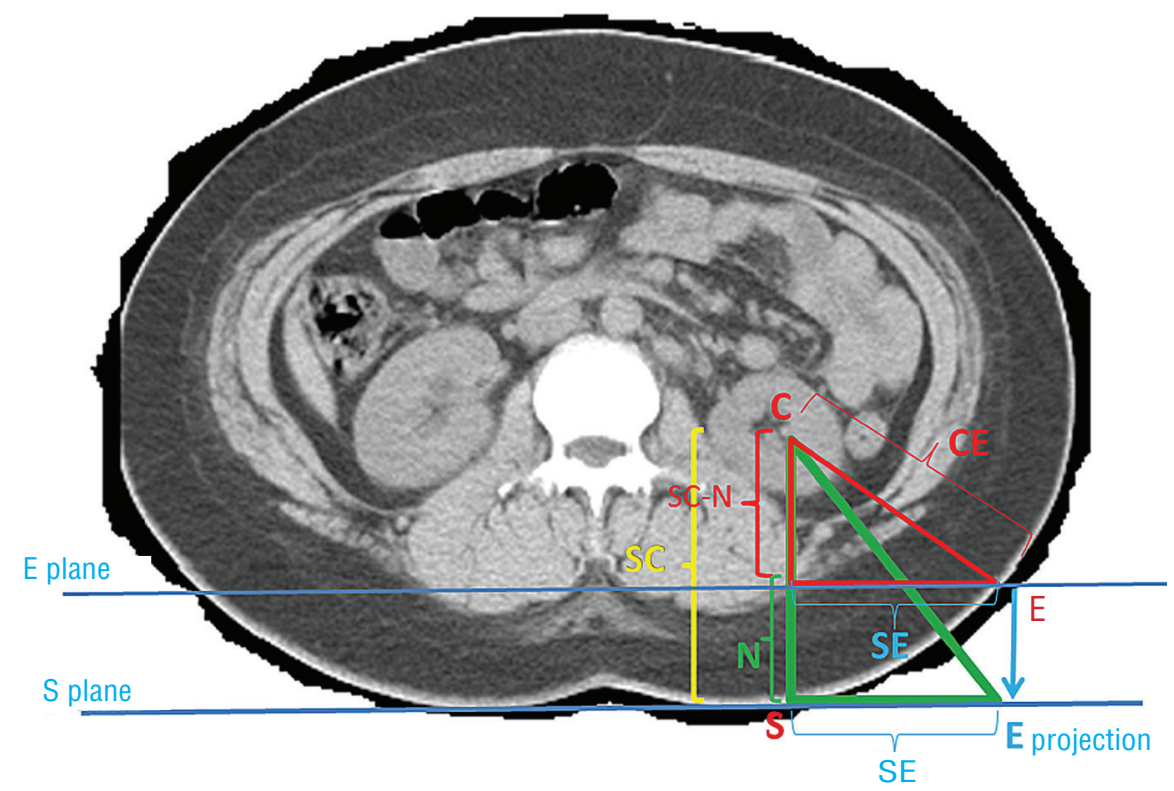


RESULTS

The mean Skin-to-Calyx Distance obtained by dorsal decubitus CT was $8.40( \pm 2.06 \mathrm{~cm})$, by ventral decubitus CT was $8.32( \pm 1.95 \mathrm{~cm})$ and by ventral decubitus US was $6.74( \pm 1.68 \mathrm{~cm})$ (Table-1). These measures showed a significant difference ( $p$ $<0.001$ ). Multiple comparisons tests showed that this difference occurred between the US and dorsal $\mathrm{CT}$ and US and ventral CT. The comparison between the CT groups (CT in DD versus CT in VD) did not show a statistically significant difference, except in the lower posterior right calyx $(p=0.002)$. The results obtained are represented in Figure-3.

The BMI was $28.66( \pm 4.66) \mathrm{Kg} / \mathrm{m}^{2}$, abdominal circumference at the level of the xiphoid process was $96.09( \pm 12.32 \mathrm{~cm})$ and at the level of the umbilicus scar was $100.79( \pm 14.66 \mathrm{~cm})$ (Table-2).

\section{DISCUSSION}

Despite the improvement in the general health and social conditions of the population as well as the advances made in preventive medicine over recent decades, we still find people with large renal calculi. The best treatment for these stones is PCNL $(2,5)$. The success of this surgery, with complete removal of the stones and small risk of complications, depends, among other factors, on the correct choice of the calyx for the puncture and on the correct puncture on the center of the calyx chosen. The correct percutaneous access used to attain the appropriate access to the calculus continues to constitute a challenge for urologists, because the guidance used for the puncture is given by fluoroscopy in one single plane. The chance of getting it right depends much more on the surgeon's expe-

Table 1 - Skin-to-Calix Distance (SCD) with mean values and standard deviations for CT and US measurements. The last line corresponds to all the calices together.

\begin{tabular}{lcccccc}
\hline & \multicolumn{2}{c}{ Dorsal CT } & \multicolumn{2}{c}{ Ventral CT } & \multicolumn{2}{c}{ Ventral US } \\
\hline Posterior calyx & Mean & $\begin{array}{c}\text { Standard } \\
\text { deviation }\end{array}$ & Mean & $\begin{array}{c}\text { Standard } \\
\text { deviation }\end{array}$ & Mean & $\begin{array}{c}\text { Standard } \\
\text { deviation }\end{array}$ \\
\hline Upper R & 7.85 & 1.77 & 8.08 & 1.83 & 6.05 & 1.49 \\
Intermed. R & 8.23 & 1.84 & 7.96 & 1.90 & 6.42 & 1.46 \\
Lower R & 9.32 & 2.07 & 8.65 & 2.02 & 6.87 & 1.47 \\
Upper L & 7.79 & 1.92 & 7.94 & 1.80 & 6.58 & 1.70 \\
Intermed. L & 8.32 & 1.96 & 8.15 & 1.91 & 6.96 & 1.69 \\
Lower L & 9.25 & 2.30 & 9.13 & 2.06 & 7.55 & 1.90 \\
All & 8.40 & 2.06 & 8.32 & 1.95 & 6.74 & 1.68 \\
\hline
\end{tabular}

The abdominal circumference at the level of the xiphoid process and at the level of the umbilicus presented a good to excellent correlation with the BMI (Pearson's Correlation Coefficient, $r=$ 0.763 and $r=0.865$, respectively, $p \leq 0.001$ for both correlations) as demonstrated in Figure-4A and B.

The skin-to-calyx distance of the lower calices of the right and left kidneys presented a moderate to good correlation with the BMI (Pearson's Correlation Coefficient, $r=0.540$ and $r=0.576$ respectively, $\mathrm{p} \leq 0.001$ for both correlations) as demonstrated in Figure-5A and B. rience than on rational calculation. Various devices have been created to meet this challenge, from the robot system devised by Cadeddu et al. (13) and modified by John Bauer et al. (14) for remote-controlled surgery, to simple manual plates (15). All of them use a system of acquisition of the angle and depth of penetration of the puncture needle based on two image acquisitions from the $\mathrm{C}$ arm of the fluoroscopy at the moment of the surgery: 0 and 30 degrees or 0 and 90 degrees.

The renal puncturometer, which is the device we have developed, uses the distance of the 
Figure 3 - Mean skin-to-calyx distance (SCD) for each calyx. Multiple comparisons between the groups of exams.
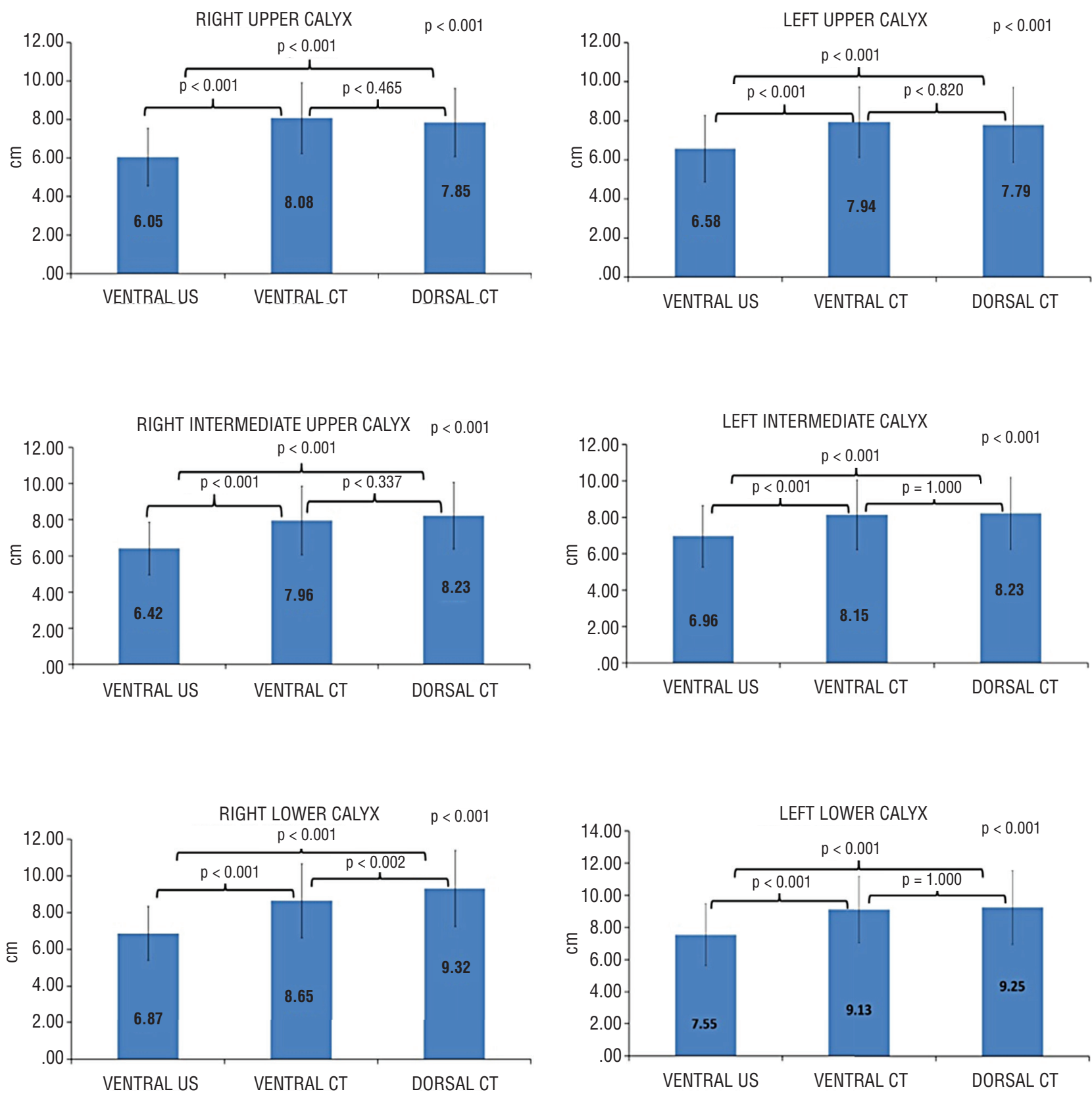

depth from the skin to the target calyx (SCD). There are various studies using CT in the dorsal and ventral positions for the localization of intraperitoneal organs, especially the colon. Ball et al. (16) studied the position of the internal organs by CT in both dorsal and ventral positions, in order to mark

them out for radiotherapy. Those authors observed that when patients are in the ventral position, the kidney is ventrally displaced by 1 to $5 \mathrm{~cm}$. We have found no study which has attempted to access this distance with the patient in position for percutaneous nephrolithotripsy. 
Table 2 - Mean, standard deviation, minimum and maximum of BMI and abdominal circumference.

\begin{tabular}{lllll}
\hline & Mean & Standard deviation & Minimum & Maximum \\
\hline BMI Kg/m² & 28.66 & 4.66 & 19.02 & 41.06 \\
Xiphoid appendix (cm) & 96.09 & 12.32 & 63.00 & 130.00 \\
Umbilical scar (cm) & 100.79 & 14.66 & 62.00 & 132.00 \\
\hline
\end{tabular}

Figure 4 - Correlation of BMI with the abdominal circumference at the level of the xiphoid process $(A)$ and at the level of the umbilicus (B).
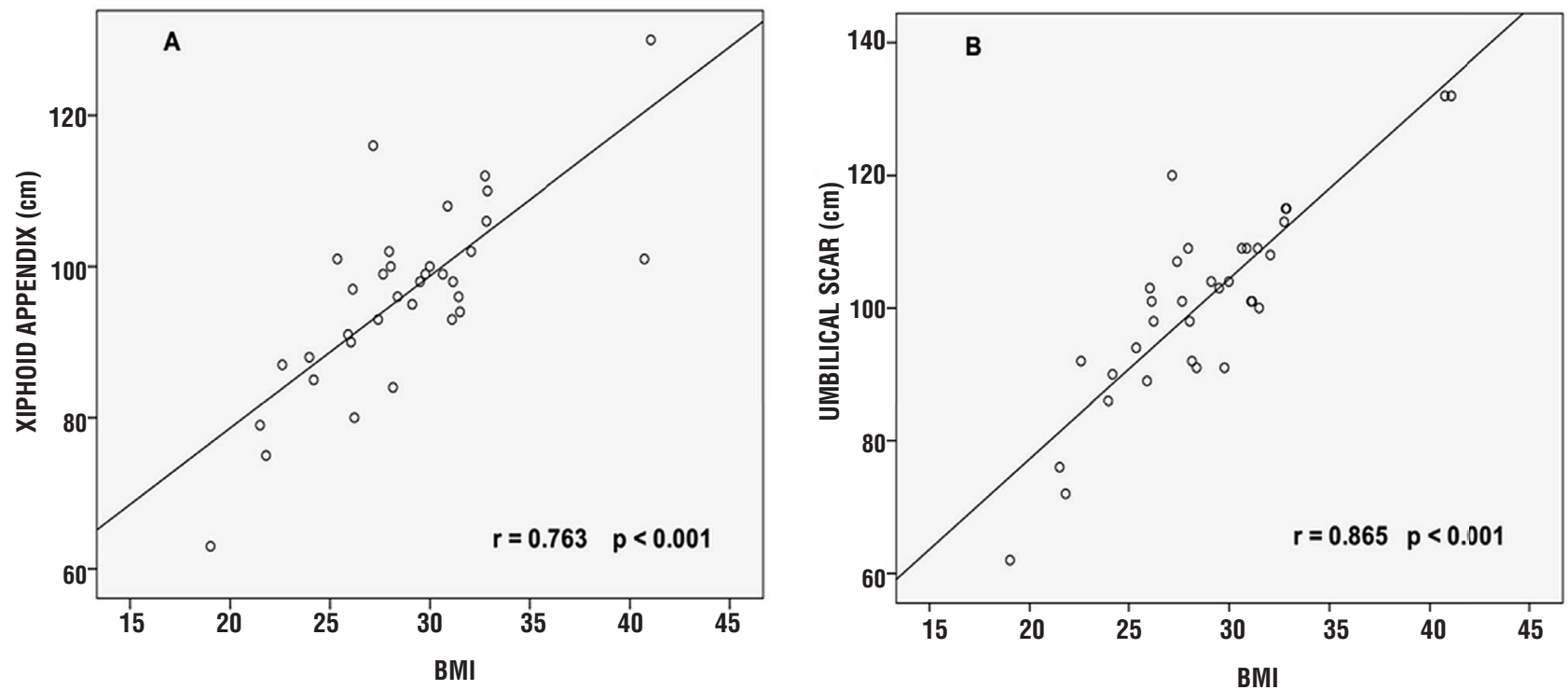

Figure 5 - Correlation of BMI with the skin-to-calyx distance of the lower calices of the right (A) and left kidneys (B).
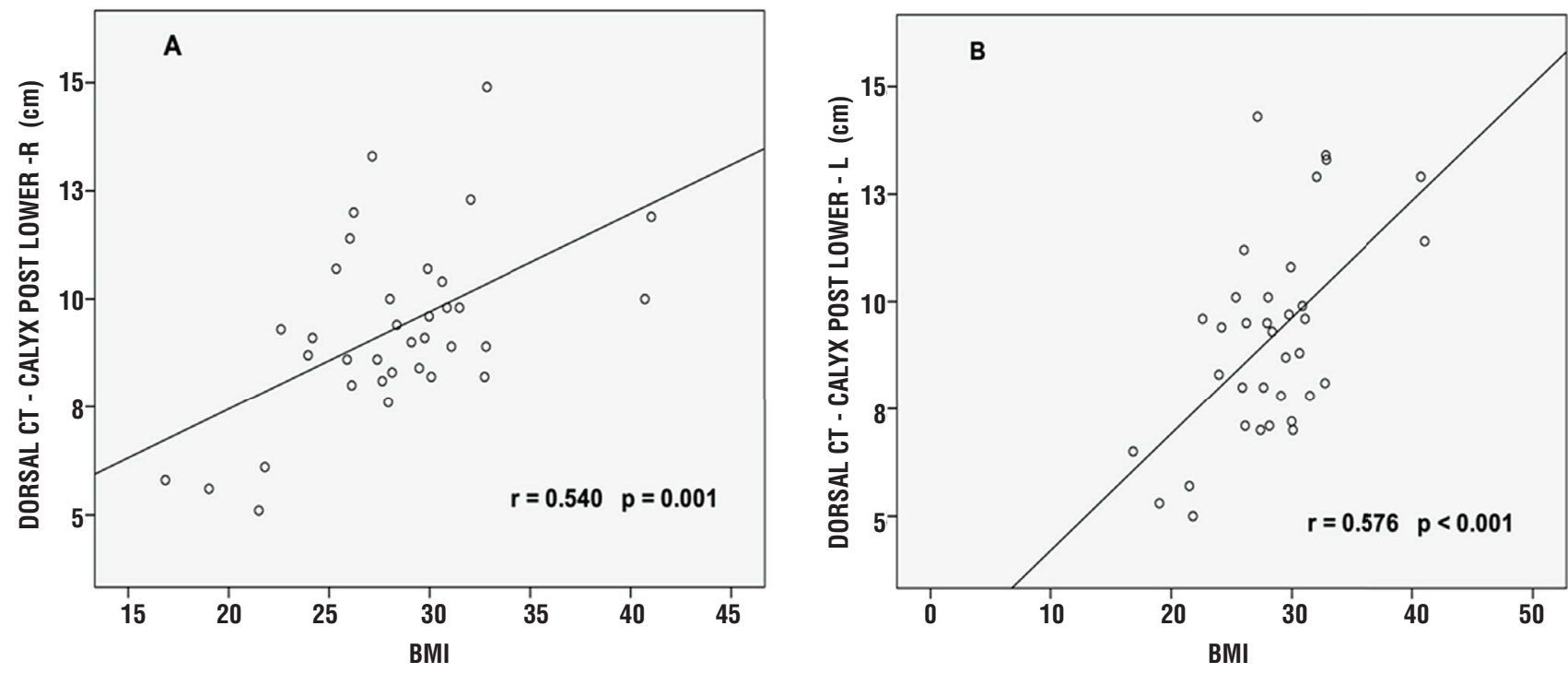

$\mathbf{R}=$ Right; $\mathbf{L}=$ left. 
Azhar et al. (17), in a study using tomography in the dorsal and ventral position to assess the relationship between the internal organs in relation to the trajectory of the needle in calyx puncture, observed that in the ventral position, the distance from the lower calyx to the skin at the point at which the needle enters is reduced from 9 to $7.6 \mathrm{~cm}$. Duty et al. (18) performed a similar study without the use of bolster. Comparing prone to ventral position, he observed a reduction in the distance of $2.57 \mathrm{~cm}$ in the right kidney and $1.83 \mathrm{~cm}$ in the left kidney. These assessments were of an oblique line, represented by the hypotenuse of our triangle (Figure-1). In our study it is a perpendicular line, represented by the vertical side of our triangle (Figure-1). This measurement in the CT exam varied very little between the dorsal and ventral decubitus positions. In the upper calyx this distance increased, on average, $0.24 \mathrm{~cm}$ in the right kidney and $0.17 \mathrm{~cm}$ in the left kidney, while in the lower calyx it diminished, on average, $0.77 \mathrm{~cm}$ in the right kidney and $0.22 \mathrm{~cm}$ in the left kidney. By virtue of the law of gravity it is expected that in the ventral decubitus position the kidney should move away from the spine, thus increasing the distance between the kidney and the dorsal wall, as was in fact found in the study of Ball et al. (16). The reduction of this distance, in the lower calyx, in our study, is probably due to the bolster placed on the abdomen, as happens in percutaneous nephrolithotripsy procedures. There was no statistical difference in the comparison of these distances under these conditions, except for the right lower calyx. For the right lower calyx we were not able to determine which CT exam (DD or VD) provides the most appropriate measurement. We hope to be able to answer that question after further studies.

There was a significant difference of the SCD in the CT both in the dorsal and ventral decubitus as compared with the US exam in the ventral decubitus, which may be due to the technical differences between the methods employed, or even to occasional pressure exercised by the ultrasonography operator on the skin. The distance obtained on the US should not, therefore, be used for the calculation of the angle of the needle's entry.
Although normally there is a correlation between BMI and the abdominal circumferences, there are people whose measurements are far from standard. As the correlation between BMI and the skin-to-calyx distance is less precise, we do not recommend its use in the determination of SCD.

\section{CONCLUSIONS}

The skin-to-calyx distances obtained by $\mathrm{CT}$ in the ventral and dorsal decubitus positions present no significant differences and both positions may be used indifferently for the calculation of the angle of entry of the needle during percutaneous surgery, in all calices, except for the lower calyx of the right kidney.

The skin-to-calyx distance obtained by the US exam cannot be used for the calculation of the angle of entry of the needle in percutaneous surgery.

A rule for the correlation between BMI and the skin to calyx distance could not be determined.

\section{ABBREVIATIONS}

$$
\begin{aligned}
& \mathrm{Kg}=\text { milogram } \\
& \mathrm{m}=\text { meter } \\
& \mathrm{cm}=\text { centimeter } \\
& \%=\text { percent } \\
& \mathrm{p}=\text { probability } \\
& \mathrm{r}=\mathrm{rho} \text { (Pearson's correlation coefficient) } \\
& \text { ESWL = extracorporeal shockwave lithotripsy } \\
& \mathrm{PCNL}=\text { percutaneous nephrolithotripsy } \\
& \mathrm{SCD}=\text { skin-to-calyx distance } \\
& \mathrm{CT}=\text { computerized tomography } \\
& \mathrm{BMI}=\text { body mass index } \\
& \mathrm{US}=\text { ultrasound } \\
& \mathrm{DD}=\text { dorsal decubitus } \\
& \text { VD = ventral decubitus }
\end{aligned}
$$

\section{CONFLICT OF INTEREST}

None declared.

\section{REFERENCES}

1. Tolon M, Miroglu C, Erol H, Tolon J, Acar D, Bazmanoglu E, et al.: A report on extracorporeal shock wave lithotripsy results on 1,569 renal units in an outpatient clinic. J Urol. 1991; 145: 695-8. 
2. Yuhico MP, Ko R: The current status of percutaneous nephrolithotomy in the management of kidney stones. Minerva Urol Nefrol. 2008; 60: 159-75.

3. Segura JW, Patterson DE, LeRoy AJ, Williams HJ Jr, Barrett $\mathrm{DM}$, Benson RC Jr, et al.: Percutaneous removal of kidney stones: review of 1,000 cases. Urol. 1985; 134: 1077-81.

4. Patel U, Hussain FF: Percutaneous nephrostomy of nondilated renal collecting systems with fluoroscopic guidance: technique and results. Radiology. 2004; 233: 226-33.

5. Preminger GM, Assimos DG, Lingeman JE, Nakada SY, Pearle MS, Wolf JS Jr, et al.: Chapter 1: AUA guideline on management of staghorn calculi: diagnosis and treatment recommendations. J Urol. 2005; 173: 1991-2000.

6. Miller NL, Matlaga BR, Lingeman JE: Techniques for fluoroscopic percutaneous renal access. J Urol. 2007; 178: 15-23.

7. Bird VG, Fallon B, Winfield HN: Practice patterns in the treatment of large renal stones. J Endourol. 2003;17: 355-63.

8. Lee CL, Anderson JK, Monga M: Residency training in percutaneous renal access: does it affect urological practice? J Urol. 2004; 171: 592-5.

9. Watterson JD, Soon S, Jana K: Access related complications during percutaneous nephrolithotomy: urology versus radiology at a single academic institution. J Urol. 2006; 176: 142-5.

10. Sampaio FJ, Zanier JF, Aragão AH, Favorito LA: Intrarenal access: 3-dimensional anatomical study. J Urol. 1992; 148: 1769-73.

11. Dawson B, Trapp RG: Bioestatística Básica e Clínica, 3th ed. Rio de Janeiro, Mc Graw Hill. 2003; pp. 42-3.

12. Maroco J: Análise estatística com utilização do SPSS, 3th ed. Lisboa, Sílabo. 2007; pp. 267-83.
13. Cadeddu JA, Bzostek A, Schreiner S, Barnes AC, Roberts WW, Anderson JH, et al.: A robotic system for percutaneous renal access. J Urol. 1997; 158: 1589-93.

14. Bauer J, Lee BR, Stoianovici D, Bishoff JT, Micali S, Micali $F$, et al.: Remote percutaneous renal access using a new automated telesurgical robotic system. Telemed J E Health. 2001; 7: 341-6.

15. Li X, Liao S, Yu Y, Dai Q, Song B, Li L: Stereotactic localisation system: a modified puncture technique for percutaneous nephrolithotomy. Urol Res. 2012; 40: 395-401.

16. Ball WS, Wicks JD, Mettler FA Jr.: Prone-supine change in organ position: CT demonstration. AJR Am J Roentgenol. 1980; 135: 815-20.

17. Azhar RA, Szymanski KM, Lemercier E, Valenti D, Andonian S, Anidjar M: Visceral organ-to-percutaneous tract distance is shorter when patients are placed in the prone position on bolsters compared with the supine position. J Endourol. 2011; 25: 687-90.

18. Duty B, Waingankar N, Okhunov Z, Ben Levi E, Smith A, Okeke Z: Anatomical variation between the prone, supine, and supine oblique positions on computed tomography: implications for percutaneous nephrolithotomy access. Urology. 2012; 79: 67-71.
Correspondence address: Chen Jen Shan, MD

Rua Castro Alves, 601, AP 71 Sao Paulo, SP, 01532-001, Brazil Telephone: +55 11 99122-6263

E-mail: c_jen_shan@yahoo.com 